\title{
White Matter Tract-Cognitive Relationships in Children with High-Functioning Autism Spectrum Disorder
}

\author{
Yoko Kato ${ }^{1}$, Kuriko Kagitani-Shimono ${ }^{1,2,3} \bowtie$, Junko Matsuzaki ${ }^{1,2}$, Ryuzo Hanaie ${ }^{1}$, Tomoka Yamamoto ${ }^{2}$, \\ Koji Tominaga ${ }^{1,2,3}$, Yoshiyuki Watanabe ${ }^{4}$, Ikuko Mohri ${ }^{1,2,3}$, and Masako Taniike ${ }^{1,2,3}$ \\ ${ }^{1}$ United Graduate School of Child Development, Osaka University, Osaka, Japan \\ ${ }^{2}$ Molecular Research Center for Children's Mental Development, Osaka University Graduate School of Medicine, Osaka, Japan \\ ${ }^{3}$ Department of Pediatrics, Osaka University Graduate School of Medicine, Osaka, Japan \\ ${ }^{4}$ Department of Diagnostic and Interventional Radiology, Osaka University Graduate School of Medicine, Osaka, Japan
}

Objective The purpose of the present study was to clarify the relationship between white matter tracts and cognitive symptoms in children with high-functioning autism spectrum disorder (ASD).

Methods We examined the cognitive functions of 17 children with high-functioning ASD and 18 typically developing (TD) controls and performed diffusion tensor imaging (DTI) tractography. We compared the results between the groups and investigated the correlations between the cognitive scores and DTI parameters within each group.

Results The Comprehension scores in the ASD group exhibited a positive correlation with mean diffusivity (MD) in the forceps minor (F minor). In the TD group, the Comprehension scores were positively correlated with fractional anisotropy (FA) in the right inferior fronto-occipital fasciculus (IFO) and left anterior thalamic radiation (ATR), and negatively correlated with MD in the left ATR, radial diffusivity (RD) in the right IFO, and RD in the left ATR. Additionally, a positive correlation was observed between the Matching Numbers scores and MD in the left uncinate fasciculus and F minor, and RD in the F minor. Furthermore, the Sentence Questions scores exhibited a positive correlation with $\mathrm{RD}$ in the right inferior longitudinal fasciculus. Relative to TD controls, the specific tract showing a strong correlation with the cognitive scores was reduced in the ASD group.

Conclusion Our findings indicate that white matter tracts connecting specific brain areas may exhibit a weaker relationship with cognitive functions in children with ASD, resulting in less efficient cognitive pathways than those observed in TD children.

Psychiatry Investig 2019;16(3):220-233

Key Words High-function autism, Diffusion tensor imaging, White matter, Cognition.

\section{INTRODUCTION}

Autism spectrum disorder (ASD) is a neurodevelopmental disorder characterized by impairments in social interaction, repetitive behavior, and communication difficulties. ${ }^{1}$ Worldwide, the prevalence of ASD is approximately $1.0 \%{ }^{2}$ Several studies have identified impairments in various cognitive functions in individuals with ASD, including language comprehension, ${ }^{3}$ planning, ${ }^{4-6}$ and working memory. ${ }^{7}$ These cognitive

Received: May 2, 2018 Revised: November 3, 2018

Accepted: January 16, 2019

$\triangle$ Correspondence: Kuriko Kagitani-Shimono, MD, PhD

United Graduate School of Child Development, Osaka University, 2-2 Yamadaoka, Suita, Osaka 565-0871, Japan

Tel: +81-6-6879-3863, Fax: +81-6-6879-3863

E-mail: kuriko@ped.med.osaka-u.ac.jp

(a) This is an Open Access article distributed under the terms of the Creative Commons Attribution Non-Commercial License (https://creativecommons.org/licenses/by$\mathrm{nc} / 4.0$ ) which permits unrestricted non-commercial use, distribution, and reproduction in any medium, provided the original work is properly cited. difficulties have been reported in individuals with high-functioning ASD as well as in those with low-functioning ASD.

Children with high-functioning ASD exhibit specific profiles when assessed using the Wechsler Intelligence Scale for Children (WISC) - the most commonly used battery for the assessment of intelligence. ${ }^{8}$ Many children with high-functioning ASD exhibit strength on the Perceptual Reasoning Index (PRI), attaining relatively higher scores on Matrix Reasoning than on other components, and weakness on the Working Memory Index (WMI) and Processing Speed Index (PSI), attaining relatively lower scores on Coding. Although their scores on the Verbal Comprehension Index (VCI) usually match those of typically developing (TD) children, children with high-functioning ASD tend to exhibit high Similarities sub-scores and low Comprehension sub-scores. ${ }^{9-11}$ In a previous study, correlation analyses revealed that low scores on Coding and Comprehension were correlated with poor communication ability 
and reciprocal social interaction, as assessed using the Autism Diagnostic Observation Schedule (ADOS).$^{10}$ In contrast, higher scores on the VCI and WMI were correlated with higher scores on the Adaptive Communication Abilities portion of the Vineland Adaptive Behavior Scales..$^{10}$ These findings suggest that the cognitive impairments identified using the WISC are associated with behavior and influence the daily life among individuals with ASD.

The Das-Naglieri Cognitive Assessment System (DN-CAS) is designed to assess four cognitive processes, namely Planning, Attention, Simultaneous, and Successive (PASS). ${ }^{12,13}$ This battery is used to support the diagnosis and classification of various neurological conditions (i.e., learning disabilities, attention-deficit/hyperactivity disorder, cognitive disabilities, giftedness, traumatic brain injury, serious emotional disturbance). The DN-CAS tests for personal strengths and weaknesses, evaluates the age-appropriateness of cognitive abilities, and predicts achievement. ${ }^{14}$ In particular, the Planning score is useful for assessing executive function, which cannot adequately be assessed using the WISC alone. ${ }^{15}$ According to one previous study that utilized the DN-CAS, individuals with ASD tend to score low on the Planning portion of the test. ${ }^{16}$

Recent advancements in neuroimaging techniques have enabled the assessment of fine brain structures using magnetic resonance imaging (MRI). Diffusion tensor imaging (DTI) is among the most useful techniques, as this method can be used to visualize anatomical connections between different brain regions and to quantify the microstructural integrity of specific white matter tracts. ${ }^{17-19}$ The four main parameters for DTI are fractional anisotropy (FA), mean diffusivity (MD), axial diffusivity $(\mathrm{AD})$, and radial diffusivity $(\mathrm{RD})$. FA represents an overall measure of white matter microstructural integrity and is sensitive to myelination, axon diameter, fiber density, and fiber coherence. ${ }^{20-23} \mathrm{MD}$ measures overall randomized water motion and is sensitive to alteration of brain tissues. ${ }^{24,25}$ $\mathrm{AD}$ measures axonal integrity and is sensitive to axonal damage. ${ }^{26} \mathrm{RD}$ measures myelin integrity, is susceptible to de- or dysmyelination, and is also affected by changes in axonal diameter or density. ${ }^{22,26-28}$

The two most frequently used methods for analyzing DTI data include the region-of-interest (ROI) method ${ }^{29,30}$ and TractBased Spatial Statistics. ${ }^{31}$ However, previous DTI studies utilizing these methods have yielded incongruent results. Such studies have reported that FA values for individuals with ASD were both lower and higher than those of controls-and that $\mathrm{RD}$ values were higher than those of controls-in the following white matter tracts: superior longitudinal fasciculus (SLF), inferior longitudinal fasciculus (ILF), inferior fronto-occipital fasciculus (IFO), uncinate fasciculus (UNC), anterior thalamic radiation (ATR), corpus callosum (CC), forceps minor
(F minor), and forceps major (F major). ${ }^{32-37}$ These tracts are significantly associated with various aspects of cognition in healthy individuals; for example, SLF is associated with language and processing speed, ${ }^{38,39}$ ILF with auditory comprehension ${ }^{40}$ IFO with semantic memory and processing speed, ${ }^{38,41}$ UNC with auditory-verbal memory, ${ }^{42}$ ATR with executive function and working memory, ${ }^{38,43} \mathrm{~F}$ minor with working memory and executive function, ${ }^{43,44}$ and $\mathrm{F}$ major with visuospatial cognition. ${ }^{45}$

In addition, several studies have investigated the correlation between white matter abnormalities and the severity of ASD. Alexander et al ${ }^{46}$ reported that $\mathrm{FA}$ in the $\mathrm{CC}$ exhibited a positive correlation with Processing Speed on the WISC in individuals with ASD. Subsequent studies revealed that FA in the right ATR and right UNC exhibited a negative correlation with the total score on the Social Responsiveness Scale. ${ }^{32}$ FA in the bilateral ILF, right IFO, and F major exhibited a negative correlation with the social score on the ADOS, while FA in the right ILF and right IFO exhibited a negative correlation with the social score on the Autism Diagnostic Interview, Revised (ADIR). ${ }^{34} \mathrm{FA}$ in the bilateral IFO and $\mathrm{F}$ major exhibited a negative correlation with the communication score on the ADOS, ${ }^{34,47}$ while FA in the left SLF and left IFO exhibited a negative correlation with the communication score on the ADI-R. ${ }^{34}$

Collectively, these findings suggest that individuals with ASD exhibit structural abnormalities in the white matter tracts of the brain and that these abnormalities are associated with the severity of autism and cognitive deficits. However, in these previous studies, the age of included individuals varied widely, ranging from childhood to adulthood. Especially during childhood, myelination of axons in the human brain plays an important role in the maturation of cognitive functions. ${ }^{48}$ According to the developmental trajectory, the time sequence of myelin maturation differs from area to area, and slow-maturing frontal tracts such as the IFO, orbitofrontal callosum, and cingulum exhibit peak levels of myelination during the thirties. ${ }^{49}$ However, only one previous study focused on schoolaged children with ASD when examining the correlation between microstructural abnormalities and behaviors, as assessed using the WISC, Social Communication Questionnaire, and Sensory Profile. ${ }^{35}$ Furthermore, few research groups have investigated and compared the detailed correlations between subtest scores on the WISC or DN-CAS and white matter tracts in children with ASD and their TD counterparts.

Therefore, in the present study, we focused on children in late childhood and early adolescence, who were still in the immature stages of myelin development. Our aim was to investigate the relationship between cognitive functions and the microstructural integrity of white matter tracts in children with ASD. We hypothesized that white matter characteristics would 
be related to cognitive impairments in children with ASD.

To achieve our aim, we first examined differences in cognitive functions between children with ASD and age-, sex-, handedness-, and IQ-matched TD controls using the WISC-IV and DN-CAS. Second, using the ROI method, we performed DTI tractography to extract key white matter tracts for cognition and compared their parameters between the groups. Third, we investigated the correlations between cognitive functions and DTI parameters within each group.

\section{METHODS}

\section{Participants}

Seventeen boys with ASD (mean age: $12.0 \pm 1.5$ years; range: 9-14 years) and 18 TD boys (mean age: $11.9 \pm 1.6$ years; range: 9-14 years) participated in the present study. Participants with ASD and TD controls were matched according to age and Full-Scale Intelligence Quotient (FSIQ: Table 1). All were confirmed to be right-handed using the Edinburgh Handedness Inventory. ${ }^{50}$ Written informed consent was obtained from all participants and their parents. The present study was conducted in accordance with the Declaration of Helsinki and was approved by the Institutional Review Board of our hospital (12168-9).

\section{Children with ASD}

Children with ASD were recruited from the Pediatric Department of our hospital and diagnosed by expert pediatric neurologists based on criteria detailed in the Diagnostic and Statistical Manual of Mental Disorders, Fifth Edition. ${ }^{1}$ Diagnoses were further confirmed using the Autism Diagnostic Observation Schedule-Generic (ADOS-G) ${ }^{51}$ Children with ASD who had a history of seizures, head injury, and those with genetic disorders were excluded. Two children with ASD were undergoing treatment with methylphenidate at the time of the study.

\section{TD children}

TD children were recruited as volunteers through adver-

Table 1. Participant characteristics

\begin{tabular}{lccllll}
\hline & \multicolumn{2}{c}{ ASD $(\mathrm{N}=17)$} & & \multicolumn{2}{c}{ TD $(\mathrm{N}=18)$} & p-value \\
\cline { 2 - 3 } & Mean & SD & & Mean & SD & \\
\hline Age (years) & 12.0 & 1.5 & & 11.9 & 1.6 & 0.915 \\
FSIQ & 105.12 & 13.24 & & 109.94 & 12.77 & 0.280 \\
Gender (male) & $17(100 \%)$ & & $18(100 \%)$ & 1.000 \\
Handedness (right) & $17(100 \%)$ & & $18(100 \%)$ & 1.000 \\
\hline
\end{tabular}

ASD: autism spectrum disorder, TD: typically developing, SD: standard deviation, FSIQ: Full-Scale Intelligence Quotient tisements in neighboring cities. TD children had no history of neurological, psychiatric, or developmental disorders and had not received special education support. They were also confirmed to have no ASD traits using the Japanese version of the Autistic Screening Questionnaire. ${ }^{52,53}$

\section{Intelligence quotient and cognitive abilities assessment}

Intelligence Quotient (IQ) was assessed using the WISCFourth Edition (WISC-IV). In the present study, to ensure average intelligence and minimize the effects of IQ on cognitive assessment, we selected participants with an FSIQ $>85$.

The WISC-IV comprises 10 core and five supplemental subtests designed to assess intellectual abilities based on the following four main cognitive indices: VCI, PRI, WMI, and PSI. The VCI includes the following five subsets: Similarities, Vocabulary, Comprehension, Information, and Word Reasoning. The PRI includes Block Design, Picture Concepts, Matrix Reasoning, and Picture Completion. The WMI includes Digit Span, Letter-Number Sequencing, and Arithmetic. The PSI includes Coding, Symbol Search, and Cancellation. All index scores have an average of 100 and a standard deviation of 15 . All subtest scores have an average of 10 and a standard deviation of 3 . Higher scores on this assessment battery are associated with better cognitive functioning. ${ }^{54,55}$

The DN-CAS comprises 12 subtests designed to assess cognitive processes including PASS. ${ }^{13,56}$ Planning includes Matching Numbers, Planned Code, and Planned Connections. Attention includes Expressive Attention, Number Detection, and Receptive Attention. Simultaneous includes Nonverbal Matrices, VerbalSpatial Relations, and Figure Memory. Successive includes Word Series, Sentence Repetition, and Sentence Questions. All PASS scale scores have an average of 100 and a standard deviation of 15. All subtest scores have an average of 10 and a standard deviation of 3. ${ }^{13,56}$ Higher scores on this assessment battery are associated with better cognitive processing skills.

\section{MRI data acquisition}

All images were acquired using a 3.0-Tesla MR system (Discovery MR750w; GE Healthcare, Milwaukee, WI, USA). DTI data were acquired using a single-shot spin-echo echo-planar imaging sequence with sensitivity-encoding parallel imaging (factor of two). Diffusion-weighted images were acquired in the axial plane with 25 non-collinear directions. The imaging parameters were as follows: repetition time $(\mathrm{TR})=12,000 \mathrm{~ms}$; echo time $(\mathrm{TE})=74.3 \mathrm{~ms}$; field-of-view $=260 \times 260 \mathrm{~mm}^{2}$; matrix size $=128 \times 128$; slice thickness $=3 \mathrm{~mm}$; slice gap $=0$; number of slices $=50$; number of excitations $=1$; and diffusion-weight factor $b=1000$ and $0 \mathrm{~s} / \mathrm{mm}^{2}$. Foam pillows and cushions were used to minimize the head motion of participants. 


\section{DTI data analysis}

\section{Preprocessing}

Preprocessing of the DTI data was performed using ExploreDTI (www.exploredti.com). The motion artifacts and eddy current-induced geometric distortions were corrected, and the B-matrix was reoriented to provide the appropriate orientational information. ${ }^{57}$ To detect group differences in head movement, the three translation and rotation parameters for each participant were extracted using ExploreDTI, and statistical analysis was performed. There were no significant differences in head motion between the two groups (translation $\mathrm{X}$ : $\mathrm{p}=0.84$, translation $\mathrm{Y}: \mathrm{p}=0.96$, translation $\mathrm{Z}: \mathrm{p}=0.55$, rotation $\mathrm{X}: \mathrm{p}=0.72$, rotation $\mathrm{Y}: \mathrm{p}=0.58$, rotation $\mathrm{Z}: \mathrm{p}=0.88$ ).

\section{Tractography approach}

Tensor calculations and tractography were performed us- ing ExploreDTI. The built-in whole-brain tractography tool of ExploreDTI was employed for all data using a deterministic streamline approach, which was based on previously developed tractography algorithms. ${ }^{57-59}$ The deterministic streamline tractography parameters were as follows: seed point resolution $=2.0 \mathrm{~mm}, 2.0 \mathrm{~mm}, 3.0 \mathrm{~mm}$; seed FA threshold $=0.2$; and angle threshold $=30^{\circ}$. A multi-ROI approach ${ }^{29}$ was used to reconstruct all tracts of interest. For this multi-ROI approach, we used the "OR/SEED," "AND," and "NOT" operations to select the fiber tracts of interest. ${ }^{60}$

\section{Reconstructed tracts and DTI outcome measurements}

Seven white matter tracts were selected for tractography, including the SLF, ILF, IFO, UNC, ATR, F minor, and F major. These tracts were reconstructed in accordance with protocols described in previous publications. ${ }^{60,61}$ We then analyzed the $\mathrm{FA}, \mathrm{MD}, \mathrm{AD}$, and $\mathrm{RD}$ value of each tract. Figure 1 depicts a rep-
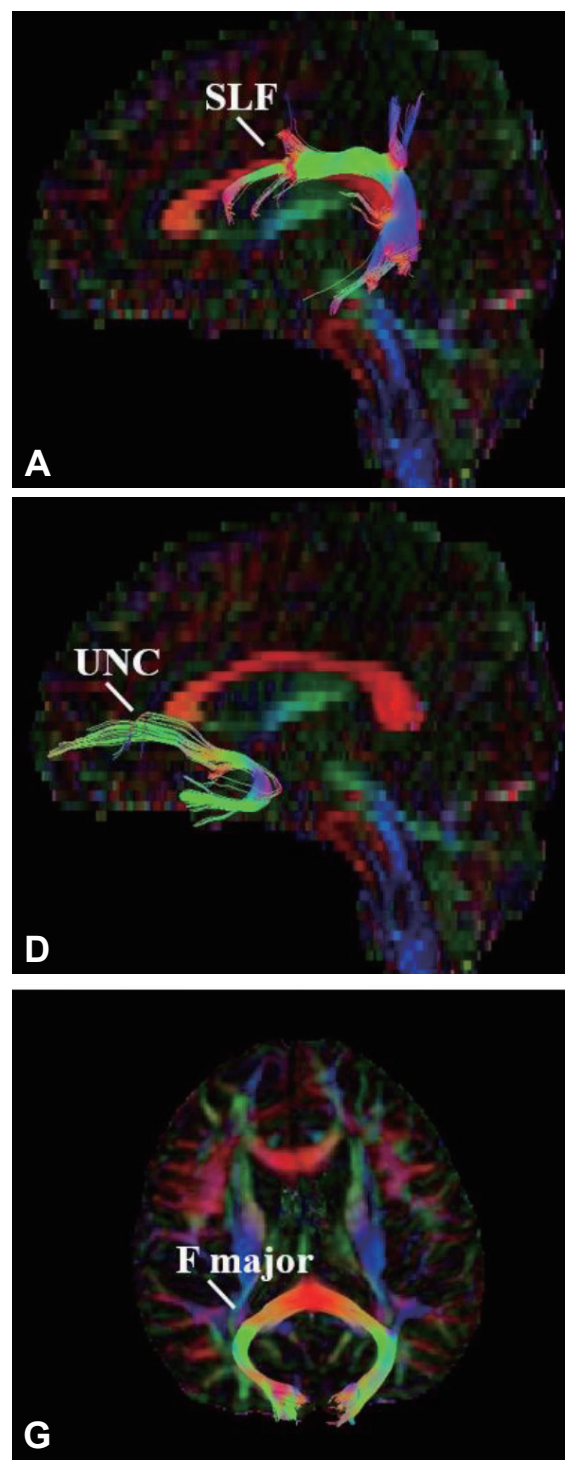
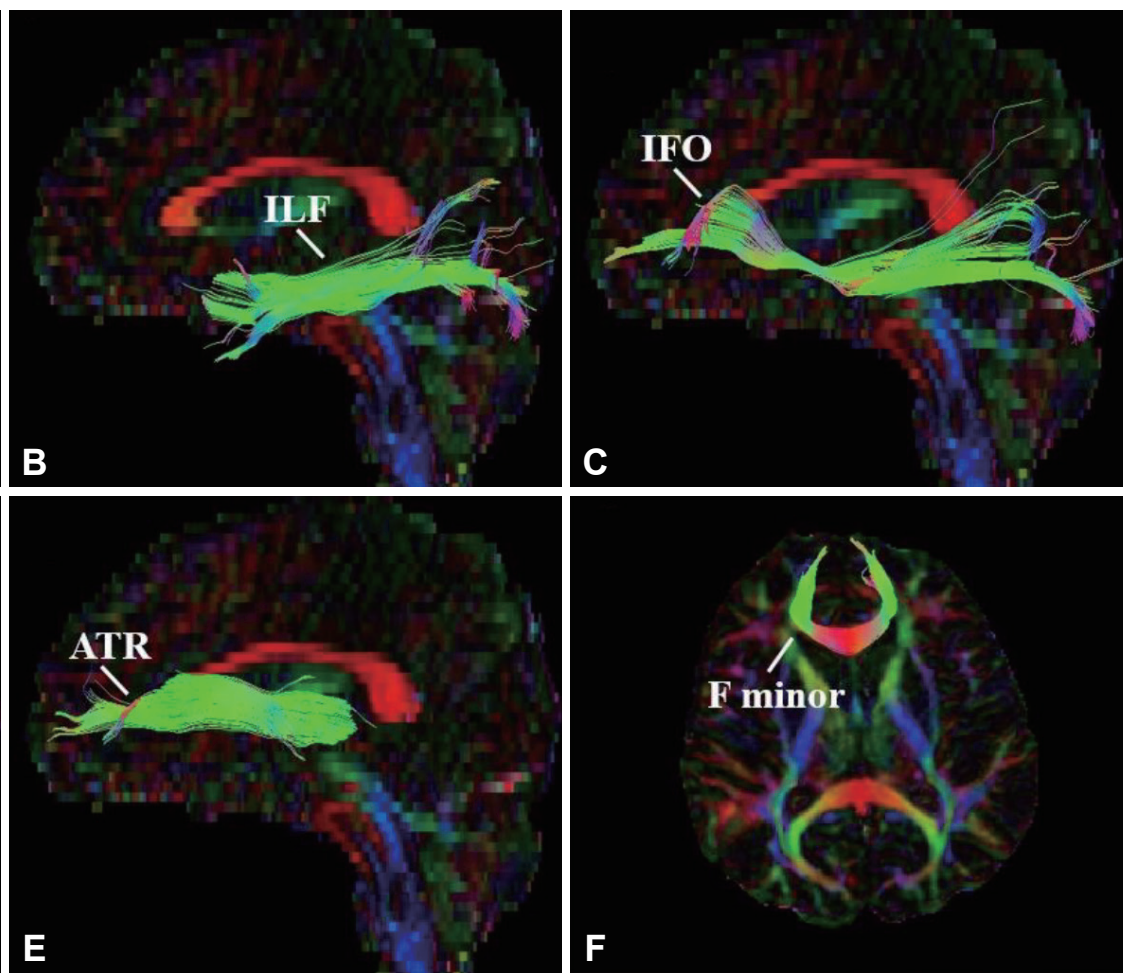

Figure 1. Reconstruction of selected tracts. A: Superior longitudinal fasciculus (SLF). B: Inferior longitudinal fasciculus (ILF). C: Inferior fronto-occipital fasciculus (IFO). D: Uncinate fasciculus (UNC). E: Anterior thalamic radiation (ATR). F: Forceps minor (F minor). G: Forceps major ( $\mathrm{F}$ major). 
resentation of the reconstructed tracts.

\section{Statistical analysis}

Statistical analyses were performed using STATA version 14.0. ${ }^{62}$ Independent-samples t-tests were used to compare the mean values for age, WISC-IV scores, and DN-CAS scores between the two groups. The tractography results were also compared using independent-samples t-tests with group (ASD/ TD) as the between-subjects factor. Within each group, Pearson's correlation coefficients were calculated between the DTI outcome measurements and the WISC-IV or DN-CAS subtest scores for which significant differences were observed to evaluate the association between microstructural integrity and cognitive function. When determining statistical significance,

Table 2. Scores on the WISC-IV

\begin{tabular}{|c|c|c|c|c|c|}
\hline & \multicolumn{2}{|c|}{$\operatorname{ASD}(\mathrm{N}=17)$} & \multicolumn{2}{|c|}{$\mathrm{TD}(\mathrm{N}=18)$} & \multirow{2}{*}{$\mathrm{p}$-value } \\
\hline & Mean & $\mathrm{SD}$ & Mean & $\mathrm{SD}$ & \\
\hline FSIQ & 105.12 & 13.24 & 109.94 & 12.77 & 0.280 \\
\hline VCI & 103.12 & 16.44 & 109.56 & 12.48 & 0.199 \\
\hline PRI & 109.35 & 11.20 & 108.39 & 12.45 & 0.812 \\
\hline WMI & 106.71 & 14.86 & 103.72 & 12.23 & 0.520 \\
\hline PSI & 94.18 & 17.44 & 105.94 & 17.23 & 0.053 \\
\hline \multicolumn{6}{|l|}{ VCI } \\
\hline Similarities & 11.65 & 2.78 & 11.56 & 2.64 & 0.921 \\
\hline Vocabulary & 10.24 & 3.31 & 11.56 & 2.94 & 0.220 \\
\hline Comprehension & 10.00 & 3.55 & 12.17 & 2.66 & $0.048^{*}$ \\
\hline Information & 11.24 & 3.21 & 10.50 & 2.23 & 0.435 \\
\hline Word reasoning & 10.82 & 2.07 & 11.83 & 2.48 & 0.201 \\
\hline \multicolumn{6}{|l|}{ PRI } \\
\hline Block design & 11.71 & 2.28 & 12.17 & 2.85 & 0.603 \\
\hline Picture concepts & 10.76 & 2.36 & 10.67 & 2.61 & 0.908 \\
\hline Matrix reasoning & 11.82 & 3.19 & 11.00 & 2.93 & 0.432 \\
\hline Picture completion & 11.65 & 2.80 & 10.50 & 2.71 & 0.227 \\
\hline \multicolumn{6}{|l|}{ WMI } \\
\hline Digit span & 10.59 & 2.98 & 11.17 & 2.18 & 0.515 \\
\hline Letter-number & 11.71 & 3.02 & 10.11 & 3.66 & 0.170 \\
\hline Sequencing & & & & & \\
\hline Arithmetic & 10.12 & 2.85 & 11.61 & 2.81 & 0.128 \\
\hline \multicolumn{6}{|l|}{ PSI } \\
\hline Coding & 8.59 & 4.32 & 11.39 & 3.43 & $0.041^{*}$ \\
\hline Symbol search & 9.53 & 2.76 & 11.11 & 3.34 & 0.138 \\
\hline Cancellation & 7.71 & 3.41 & 11.39 & 3.36 & $0.003^{* *}$ \\
\hline
\end{tabular}

${ }^{*} \mathrm{p}<0.05,{ }^{* *} \mathrm{p}<0.01$, significance level. WISC-IV: Wechsler Intelligence Scale for Children-Fourth Edition, ASD: autism spectrum disorder, TD: typically developing, SD: standard deviation, FSIQ: Full-Scale Intelligence Quotient, VCI: Verbal Comprehension Index, PRI: Perceptual Reasoning Index, WMI: Working Memory Index, PSI: Processing Speed Index
Bonferroni correction was performed for multiple comparisons $(\mathrm{p}=0.004)$.

\section{RESULTS}

\section{Between-group differences in WISC-IV and DN-CAS scores}

There were no significant differences in the WISC-IV index values between the two groups. However, the ASD group exhibited significantly lower scores than did TD controls on the following WISC-IV subscales: Comprehension $(p=0.048)$, Coding ( $\mathrm{p}=0.041)$, and Cancellation $(\mathrm{p}=0.003)$ (Table 2). Although there were no significant differences in the total DNCAS scores, the ASD group exhibited significantly poorer performance than did the TD group on the Planning $(p=0.011)$ portion of the DN-CAS and on the Matching Numbers $(\mathrm{p}=$ 0.027 ) and Sentence Questions ( $p=0.003$ ) subtests (Table 3).

\section{Between-group differences in DTI measurements}

In the ASD group, the FA value of F major was lower ( $p=$

Table 3. Scores on the DN-CAS

\begin{tabular}{|c|c|c|c|c|c|}
\hline & \multicolumn{2}{|c|}{$\operatorname{ASD}(\mathrm{N}=17)$} & \multicolumn{2}{|c|}{$\mathrm{TD}(\mathrm{N}=18)$} & \multirow{2}{*}{ p-value } \\
\hline & Mean & $\mathrm{SD}$ & Mean & $\mathrm{SD}$ & \\
\hline Total score & 103.65 & 14.71 & 111.72 & 10.06 & 0.066 \\
\hline Planning & 102.82 & 15.63 & 115.39 & 11.66 & $0.011^{*}$ \\
\hline Simultaneous & 110.59 & 15.23 & 106.28 & 16.36 & 0.426 \\
\hline Attention & 97.65 & 11.94 & 103.61 & 13.56 & 0.178 \\
\hline Successive & 99.94 & 17.30 & 109.44 & 10.80 & 0.058 \\
\hline \multicolumn{6}{|l|}{ Planning } \\
\hline Matching numbers & 10.59 & 3.24 & 12.94 & 2.75 & $0.027^{*}$ \\
\hline Planned codes & 10.18 & 3.50 & 12.17 & 3.47 & 0.101 \\
\hline Planned connections & 10.59 & 3.04 & 12.17 & 1.87 & 0.071 \\
\hline \multicolumn{6}{|l|}{ Simultaneous } \\
\hline Nonverbal matrices & 12.76 & 2.91 & 11.61 & 3.53 & 0.301 \\
\hline Verbal-spatial relations & 11.76 & 3.17 & 11.06 & 3.44 & 0.531 \\
\hline Figure memory & 10.18 & 2.56 & 10.11 & 2.59 & 0.941 \\
\hline \multicolumn{6}{|l|}{ Attention } \\
\hline Expressive attention & 9.88 & 2.67 & 10.39 & 2.30 & 0.551 \\
\hline Number detection & 10.12 & 2.62 & 11.28 & 2.82 & 0.217 \\
\hline Receptive attention & 9.06 & 2.70 & 10.17 & 3.17 & 0.275 \\
\hline \multicolumn{6}{|l|}{ Successive } \\
\hline Word series & 11.50 & 3.69 & 12.11 & 2.83 & 0.678 \\
\hline Sentence repetition & 10.41 & 3.39 & 11.11 & 2.72 & 0.505 \\
\hline Sentence questions & 7.94 & 3.47 & 11.39 & 2.77 & $0.003^{* *}$ \\
\hline
\end{tabular}

${ }^{*} \mathrm{p}<0.05,{ }^{* *} \mathrm{p}<0.01$, significance level. DN-CAS: Das-Naglieri Cognitive Assessment System, ASD: autism spectrum disorder, TD: typically developing, SD: standard deviation 
Table 4. Fractional anisotropy, mean diffusivity, axial diffusivity, and radial diffusivity values in seven white matter pathways

\begin{tabular}{|c|c|c|c|c|c|c|}
\hline & \multicolumn{2}{|c|}{$\operatorname{ASD}(\mathrm{N}=17)$} & \multicolumn{2}{|c|}{$\mathrm{TD}(\mathrm{N}=18)$} & \multirow{2}{*}{ p-value } \\
\hline & & Mean & $\mathrm{SD}$ & Mean & SD & \\
\hline \multirow[t]{4}{*}{ SLF left } & FA & 0.46 & 0.02 & 0.46 & 0.02 & 0.759 \\
\hline & $\mathrm{MD}$ & 0.79 & 0.02 & 0.78 & 0.03 & 0.566 \\
\hline & $\mathrm{AD}$ & 1.21 & 0.04 & 1.21 & 0.04 & 0.542 \\
\hline & $\mathrm{RD}$ & 0.58 & 0.02 & 0.57 & 0.03 & 0.730 \\
\hline \multirow[t]{4}{*}{ SLF right } & FA & 0.47 & 0.02 & 0.47 & 0.03 & 0.209 \\
\hline & $\mathrm{MD}$ & 0.74 & 0.06 & 0.73 & 0.03 & 0.156 \\
\hline & $\mathrm{AD}$ & 1.15 & 0.04 & 1.14 & 0.04 & 0.449 \\
\hline & $\mathrm{RD}$ & 0.54 & 0.02 & 0.52 & 0.03 & 0.135 \\
\hline \multirow[t]{4}{*}{ ILF left } & FA & 0.44 & 0.02 & 0.44 & 0.02 & 0.739 \\
\hline & $\mathrm{MD}$ & 0.86 & 0.06 & 0.85 & 0.06 & 0.209 \\
\hline & $\mathrm{AD}$ & 1.31 & 0.04 & 1.29 & 0.03 & 0.184 \\
\hline & $\mathrm{RD}$ & 0.63 & 0.02 & 0.62 & 0.03 & 0.417 \\
\hline \multirow[t]{4}{*}{ ILF right } & FA & 0.46 & 0.03 & 0.48 & 0.02 & 0.218 \\
\hline & $\mathrm{MD}$ & 0.81 & 0.02 & 0.80 & 0.03 & 0.137 \\
\hline & $\mathrm{AD}$ & 1.26 & 0.04 & 1.25 & 0.03 & 0.457 \\
\hline & $\mathrm{RD}$ & 0.58 & 0.03 & 0.57 & 0.03 & 0.177 \\
\hline \multirow[t]{4}{*}{ IFO left } & FA & 0.46 & 0.02 & 0.47 & 0.02 & 0.208 \\
\hline & $\mathrm{MD}$ & 0.83 & 0.02 & 0.82 & 0.02 & 0.215 \\
\hline & $\mathrm{AD}$ & 1.30 & 0.04 & 1.30 & 0.03 & 0.807 \\
\hline & $\mathrm{RD}$ & 0.60 & 0.20 & 0.59 & 0.02 & 0.126 \\
\hline \multirow[t]{4}{*}{ IFO right } & FA & 0.48 & 0.02 & 0.48 & 0.02 & 0.819 \\
\hline & $\mathrm{MD}$ & 0.81 & 0.02 & 0.79 & 0.02 & 0.080 \\
\hline & $\mathrm{AD}$ & 1.28 & 0.04 & 1.26 & 0.03 & 0.086 \\
\hline & $\mathrm{RD}$ & 0.57 & 0.02 & 0.56 & 0.02 & 0.167 \\
\hline \multirow[t]{4}{*}{ UNC left } & FA & 0.41 & 0.02 & 0.41 & 0.02 & 0.508 \\
\hline & $\mathrm{MD}$ & 0.81 & 0.02 & 0.81 & 0.02 & 0.892 \\
\hline & $\mathrm{AD}$ & 1.20 & 0.02 & 1.20 & 0.02 & 0.624 \\
\hline & $\mathrm{RD}$ & 0.60 & 0.02 & 0.59 & 0.02 & 0.681 \\
\hline \multirow[t]{4}{*}{ UNC right } & FA & 0.41 & 0.03 & 0.42 & 0.02 & 0.531 \\
\hline & MD & 0.80 & 0.02 & 0.80 & 0.02 & 0.546 \\
\hline & $\mathrm{AD}$ & 1.19 & 0.03 & 1.19 & 0.02 & 0.751 \\
\hline & $\mathrm{RD}$ & 0.60 & 0.02 & 0.60 & 0.02 & 0.520 \\
\hline \multirow[t]{4}{*}{ ATR left } & FA & 0.39 & 0.02 & 0.39 & 0.03 & 0.819 \\
\hline & $\mathrm{MD}$ & 0.79 & 0.02 & 0.79 & 0.02 & 0.885 \\
\hline & $\mathrm{AD}$ & 1.14 & 0.03 & 1.14 & 0.03 & 0.669 \\
\hline & $\mathrm{RD}$ & 0.61 & 0.02 & 0.61 & 0.03 & 0.952 \\
\hline \multirow[t]{4}{*}{ ATR right } & FA & 0.40 & 0.02 & 0.40 & 0.02 & 0.925 \\
\hline & $\mathrm{MD}$ & 0.79 & 0.02 & 0.78 & 0.03 & 0.267 \\
\hline & $\mathrm{AD}$ & 1.15 & 0.03 & 1.14 & 0.04 & 0.235 \\
\hline & $\mathrm{RD}$ & 0.60 & 0.02 & 0.59 & 0.03 & 0.428 \\
\hline
\end{tabular}

Table 4. Fractional anisotropy, mean diffusivity, axial diffusivity, and radial diffusivity values in seven white matter pathways (contiued)

\begin{tabular}{|c|c|c|c|c|c|c|}
\hline & \multicolumn{2}{|c|}{$\operatorname{ASD}(n=17)$} & \multicolumn{2}{|c|}{$\mathrm{TD}(\mathrm{n}=18)$} & \multirow{2}{*}{ p-value } \\
\hline & & Mean & $\mathrm{SD}$ & Mean & $\mathrm{SD}$ & \\
\hline \multirow[t]{4}{*}{ F minor } & FA & 0.53 & 0.02 & 0.54 & 0.02 & 0.344 \\
\hline & $\mathrm{MD}$ & 0.81 & 0.02 & 0.81 & 0.02 & 0.827 \\
\hline & $\mathrm{AD}$ & 1.37 & 0.02 & 1.38 & 0.03 & 0.427 \\
\hline & $\mathrm{RD}$ & 0.53 & 0.03 & 0.52 & 0.03 & 0.507 \\
\hline \multirow[t]{4}{*}{ F major } & FA & 0.61 & 0.02 & 0.63 & 0.02 & $0.049^{*}$ \\
\hline & $\mathrm{MD}$ & 0.84 & 0.04 & 0.82 & 0.04 & 0.237 \\
\hline & $\mathrm{AD}$ & 1.54 & 0.06 & 1.54 & 0.06 & 0.996 \\
\hline & $\mathrm{RD}$ & 0.49 & 0.04 & 0.47 & 0.03 & 0.065 \\
\hline
\end{tabular}

Units of axial diffusivity and radial diffusivity are $10^{-3} \mathrm{~mm}^{2} / \mathrm{s}$ for the mean and standard deviation. ${ }^{*} \mathrm{p}<0.05$, significance level. ASD: autism spectrum disorder, TD: typically developing, SD: standard deviation, FA: fractional anisotropy, $\mathrm{MD}$ : mean diffusivity, $\mathrm{AD}$ : axial diffusivity, RD: radial diffusivity, SLF: superior longitudinal fasciculus, ILF: inferior longitudinal fasciculus, IFO: inferior fronto-occipital fasciculus, UNC: uncinate fasciculus, ATR: anterior thalamic radiation, F minor: forceps minor, F major: forceps major

0.049) than that in the TD group. However, there were no significant differences in the $\mathrm{MD}, \mathrm{AD}$, or $\mathrm{RD}$ values of any white matter tracts examined (Table 4).

\section{Correlations between the DTI measurements and WISC-IV and DN-CAS subtests}

We investigated the correlations between the DTI measurements and the subtest scores on the WISC-IV and DN-CAS. Within the TD group, the Comprehension scores on the WISCIV were positively correlated with FA in the right IFO $(r=0.71$, $\mathrm{p}=0.0009)$ and left ATR $(\mathrm{r}=0.67, \mathrm{p}=0.002)$, and negatively correlated with $\mathrm{MD}$ in the left ATR ( $\mathrm{r}=-0.75, \mathrm{p}=0.0003), \mathrm{RD}$ in the right IFO ( $\mathrm{r}=-0.66, \mathrm{p}=0.003)$, and $\mathrm{RD}$ in the left ATR ( $\mathrm{r}=$ $-0.80, p=0.0001$ ) (Table 5, Figure 2A-C, E, and F). In addition, participants' scores on the Matching Numbers subsection of the DN-CAS exhibited a significant positive correlation with $\mathrm{MD}$ in the left UNC ( $\mathrm{r}=0.65, \mathrm{p}=0.004)$ and $\mathrm{F}$ minor $(\mathrm{r}=0.67$, $\mathrm{r}=0.003)$, and with $\mathrm{RD}$ in the $\mathrm{F}$ minor $(\mathrm{r}=0.74, \mathrm{p}=0.0004)$. Furthermore, a positive correlation was observed between the scores on the Sentence Questions subsection of the DN-CAS and $\mathrm{RD}$ in the right ILF $(\mathrm{r}=0.65, \mathrm{p}=0.004)$. These results remained significant after applying Bonferroni correction (Table 6, Figure 2G-J).

Within the ASD group, only a positive correlation between the Comprehension scores on the WISC-IV and MD in F minor remained significant after Bonferroni correction $(\mathrm{r}=0.68$, $\mathrm{p}=0.003$ ) (Table 5, Figure 2D). No other significant correlations between the DTI measurements and participants' scores on any subtests were observed. In the ASD group, non-sig- 
Table 5. Correlations between the comprehension, coding, and cancellation scores on the WISC-IV and the FA, MD, AD, and RD of white matter tracts

\begin{tabular}{|c|c|c|c|c|c|c|c|c|c|c|c|c|c|}
\hline & & \multicolumn{4}{|c|}{ Comprehension } & \multicolumn{4}{|c|}{ Coding } & \multicolumn{4}{|c|}{ Cancellation } \\
\hline & & \multicolumn{2}{|c|}{$\operatorname{ASD}(\mathrm{N}=17)$} & \multicolumn{2}{|c|}{$\mathrm{TD}(\mathrm{N}=18)$} & \multicolumn{2}{|c|}{$\operatorname{ASD}(\mathrm{N}=17)$} & \multicolumn{2}{|c|}{$\mathrm{TD}(\mathrm{N}=18)$} & \multicolumn{2}{|c|}{$\operatorname{ASD}(\mathrm{N}=17)$} & \multicolumn{2}{|c|}{$\mathrm{TD}(\mathrm{N}=18)$} \\
\hline & & r value & $\mathrm{p}$ value & r value & $\mathrm{p}$ value & r value & $\mathrm{p}$ value & rvalue & $\mathrm{p}$ value & r value & $\mathrm{p}$ value & r value & $\mathrm{p}$ value \\
\hline \multirow[t]{4}{*}{$\overline{\text { SLF left }}$} & FA & -0.17 & 0.526 & 0.47 & $0.047^{*}$ & 0.25 & 0.334 & 0.11 & 0.670 & 0.08 & 0.747 & -0.13 & 0.616 \\
\hline & $\mathrm{MD}$ & 0.28 & 0.273 & -0.62 & $0.006^{*}$ & 0.15 & 0.555 & -0.31 & 0.214 & 0.25 & 0.331 & -0.08 & 0.763 \\
\hline & $\mathrm{AD}$ & 0.14 & 0.604 & -0.36 & 0.138 & 0.25 & 0.328 & -0.29 & 0.249 & 0.26 & 0.316 & -0.20 & 0.422 \\
\hline & $\mathrm{RD}$ & 0.30 & 0.246 & -0.62 & $0.006^{*}$ & 0.00 & 0.994 & -0.23 & 0.355 & 0.14 & 0.589 & 0.04 & 0.879 \\
\hline \multirow[t]{4}{*}{ SLF right } & FA & -0.02 & 0.933 & 0.53 & $0.023^{*}$ & 0.06 & 0.819 & 0.07 & 0.796 & 0.10 & 0.696 & -0.00 & 0.991 \\
\hline & $\mathrm{MD}$ & 0.28 & 0.270 & -0.52 & $0.026^{*}$ & -0.07 & 0.794 & -0.17 & 0.504 & 0.06 & 0.813 & 0.07 & 0.787 \\
\hline & $\mathrm{AD}$ & 0.25 & 0.331 & -0.20 & 0.424 & -0.08 & 0.751 & -0.19 & 0.460 & 0.08 & 0.754 & -0.00 & 0.991 \\
\hline & $\mathrm{RD}$ & 0.23 & 0.368 & -0.59 & $0.009^{*}$ & -0.04 & 0.887 & -0.10 & 0.701 & 0.03 & 0.914 & 0.10 & 0.694 \\
\hline \multirow[t]{4}{*}{ ILF left } & FA & -0.31 & 0.220 & 0.31 & 0.213 & -0.05 & 0.841 & 0.23 & 0.360 & -0.29 & 0.253 & 0.15 & 0.559 \\
\hline & $\mathrm{MD}$ & 0.14 & 0.587 & -0.45 & 0.058 & -0.43 & 0.084 & 0.02 & 0.950 & -0.39 & 0.117 & 0.13 & 0.628 \\
\hline & $\mathrm{AD}$ & -0.04 & 0.871 & -0.26 & 0.294 & -0.37 & 0.142 & 0.28 & 0.253 & -0.51 & $0.035^{*}$ & 0.37 & 0.132 \\
\hline & $\mathrm{RD}$ & 0.25 & 0.324 & -0.43 & 0.072 & -0.32 & 0.211 & -0.12 & 0.632 & -0.13 & 0.610 & -0.03 & 0.903 \\
\hline \multirow[t]{4}{*}{ ILF right } & FA & -0.08 & 0.751 & 0.56 & $0.015^{*}$ & 0.06 & 0.822 & 0.24 & 0.338 & -0.25 & 0.341 & 0.04 & 0.883 \\
\hline & $\mathrm{MD}$ & 0.37 & 0.143 & -0.47 & $0.049^{*}$ & -0.17 & 0.502 & 0.00 & 0.996 & 0.13 & 0.612 & 0.10 & 0.682 \\
\hline & $\mathrm{AD}$ & 0.29 & 0.270 & 0.00 & 0.996 & -0.06 & 0.830 & 0.33 & 0.186 & -0.12 & 0.658 & 0.26 & 0.303 \\
\hline & $\mathrm{RD}$ & 0.24 & 0.349 & -0.55 & $0.017^{*}$ & -0.17 & 0.526 & -0.14 & 0.580 & 0.23 & 0.379 & 0.01 & 0.965 \\
\hline \multirow[t]{4}{*}{ IFO left } & FA & -0.06 & 0.811 & 0.55 & $0.018^{*}$ & -0.05 & 0.856 & 0.21 & 0.393 & -0.00 & 0.987 & 0.31 & 0.217 \\
\hline & $\mathrm{MD}$ & 0.25 & 0.330 & -0.44 & 0.065 & -0.35 & 0.164 & -0.01 & 0.960 & 0.29 & 0.256 & 0.25 & 0.327 \\
\hline & $\mathrm{AD}$ & 0.24 & 0.360 & -0.01 & 0.969 & -0.35 & 0.174 & 0.13 & 0.615 & -0.23 & 0.380 & 0.52 & $0.028^{*}$ \\
\hline & $\mathrm{RD}$ & 0.17 & 0.503 & -0.56 & $0.015^{*}$ & -0.24 & 0.362 & -0.10 & 0.683 & -0.24 & 0.346 & -0.04 & 0.878 \\
\hline \multirow[t]{4}{*}{ IFO right } & FA & -0.37 & 0.141 & 0.71 & $0.0009^{* *}$ & -0.27 & 0.290 & 0.53 & $0.023^{*}$ & -0.35 & 0.170 & 0.44 & 0.071 \\
\hline & $\mathrm{MD}$ & 0.00 & 0.995 & -0.51 & $0.032^{*}$ & -0.44 & 0.080 & 0.11 & 0.678 & -0.19 & 0.474 & 0.16 & 0.524 \\
\hline & $\mathrm{AD}$ & -0.15 & 0.560 & -0.01 & 0.969 & -0.51 & $0.037^{*}$ & 0.62 & $0.006^{*}$ & -0.33 & 0.189 & 0.63 & $0.005^{*}$ \\
\hline & $\mathrm{RD}$ & 0.17 & 0.530 & -0.66 & $0.003^{* *}$ & -0.27 & 0.290 & -0.20 & 0.426 & 0.01 & 0.976 & -0.13 & 0.601 \\
\hline \multirow[t]{4}{*}{ UNC left } & FA & -0.01 & 0.978 & 0.43 & 0.072 & -0.12 & 0.641 & 0.05 & 0.838 & -0.14 & 0.587 & 0.14 & 0.579 \\
\hline & $\mathrm{MD}$ & 0.32 & 0.215 & -0.20 & 0.423 & -0.35 & 0.166 & 0.21 & 0.399 & -0.10 & 0.693 & 0.17 & 0.493 \\
\hline & $\mathrm{AD}$ & 0.35 & 0.172 & 0.18 & 0.483 & -0.52 & $0.032^{*}$ & 0.36 & 0.139 & -0.24 & 0.343 & 0.43 & 0.073 \\
\hline & $\mathrm{RD}$ & 0.22 & 0.386 & -0.31 & 0.218 & -0.16 & 0.528 & 0.12 & 0.650 & 0.01 & 0.971 & 0.04 & 0.866 \\
\hline \multirow[t]{4}{*}{ UNC right } & FA & -0.02 & 0.950 & 0.41 & 0.088 & 0.08 & 0.774 & 0.07 & 0.779 & -0.08 & 0.768 & -0.13 & 0.599 \\
\hline & $\mathrm{MD}$ & 0.14 & 0.598 & -0.42 & 0.086 & -0.38 & 0.134 & 0.06 & 0.800 & 0.84 & 0.748 & 0.15 & 0.561 \\
\hline & $\mathrm{AD}$ & 0.14 & 0.592 & -0.23 & 0.348 & -0.40 & 0.113 & 0.15 & 0.545 & 0.03 & 0.909 & 0.11 & 0.665 \\
\hline & $\mathrm{RD}$ & 0.11 & 0.671 & -0.43 & 0.073 & -0.30 & 0.246 & 0.02 & 0.942 & 0.10 & 0.840 & 0.14 & 0.575 \\
\hline \multirow[t]{4}{*}{ ATR left } & FA & -0.51 & $0.037^{*}$ & 0.67 & $0.002^{* *}$ & -0.48 & 0.053 & 0.53 & $0.024^{*}$ & -0.13 & 0.627 & 0.54 & $0.021^{*}$ \\
\hline & $\mathrm{MD}$ & 0.41 & 0.106 & -0.75 & $0.0003^{* *}$ & -0.11 & 0.670 & -0.39 & 0.107 & -0.19 & 0.470 & -0.14 & 0.582 \\
\hline & $\mathrm{AD}$ & 0.07 & 0.781 & -0.17 & 0.507 & -0.41 & 0.098 & 0.09 & 0.720 & -0.23 & 0.383 & 0.42 & 0.086 \\
\hline & $\mathrm{RD}$ & 0.52 & $0.033^{*}$ & -0.80 & $0.0001^{* *}$ & 0.13 & 0.630 & -0.50 & $0.033^{*}$ & -0.11 & 0.676 & -0.36 & 0.140 \\
\hline \multirow[t]{4}{*}{ ATR right } & FA & -0.34 & 0.181 & 0.40 & 0.099 & 0.24 & 0.351 & 0.49 & $0.040^{*}$ & -0.11 & 0.681 & 0.61 & $0.008^{*}$ \\
\hline & $\mathrm{MD}$ & 0.52 & $0.034^{*}$ & -0.56 & $0.015^{*}$ & -0.07 & 0.797 & -0.29 & 0.237 & 0.14 & 0.588 & -0.02 & 0.926 \\
\hline & $\mathrm{AD}$ & 0.26 & 0.314 & -0.40 & 0.101 & 0.11 & 0.671 & -0.08 & 0.752 & 0.05 & 0.836 & 0.40 & 0.101 \\
\hline & $\mathrm{RD}$ & 0.52 & $0.033^{*}$ & -0.56 & $0.015^{*}$ & -0.17 & 0.514 & -0.38 & 0.125 & 0.15 & 0.555 & 0.29 & 0.243 \\
\hline
\end{tabular}


Table 5. Correlations between the comprehension, coding, and cancellation scores on the WISC-IV and the FA, MD, AD, and RD of white matter tracts (contiued)

\begin{tabular}{|c|c|c|c|c|c|c|c|c|c|c|c|c|c|}
\hline & & \multicolumn{4}{|c|}{ Comprehension } & \multicolumn{4}{|c|}{ Coding } & \multicolumn{4}{|c|}{ Cancellation } \\
\hline & & \multicolumn{2}{|c|}{ ASD (N=17) } & \multicolumn{2}{|c|}{$\mathrm{TD}(\mathrm{N}=18)$} & \multicolumn{2}{|c|}{$\operatorname{ASD}(\mathrm{N}=17)$} & \multicolumn{2}{|c|}{$\mathrm{TD}(\mathrm{N}=18)$} & \multicolumn{2}{|c|}{ ASD (N=17) } & \multicolumn{2}{|c|}{$\mathrm{TD}(\mathrm{N}=18)$} \\
\hline & & rvalue & $\mathrm{p}$ value & r value & $\mathrm{p}$ value & r value & $\mathrm{p}$ value & rvalue & $\mathrm{p}$ value & rvalue & $\mathrm{p}$ value & r value & $\mathrm{p}$ value \\
\hline \multirow[t]{4}{*}{ F minor } & FA & -0.34 & 0.186 & 0.50 & $0.034^{*}$ & 0.15 & 0.561 & 0.21 & 0.410 & 0.05 & 0.840 & 0.22 & 0.375 \\
\hline & $\mathrm{MD}$ & 0.68 & $0.003^{* *}$ & -0.43 & 0.079 & -0.15 & 0.574 & 0.01 & 0.972 & 0.15 & 0.555 & 0.17 & 0.495 \\
\hline & $\mathrm{AD}$ & 0.55 & $0.022^{*}$ & 0.07 & 0.772 & -0.01 & 0.985 & 0.26 & 0.301 & 0.28 & 0.270 & 0.52 & $0.028^{*}$ \\
\hline & $\mathrm{RD}$ & 0.56 & $0.019^{*}$ & -0.53 & $0.024^{*}$ & -0.18 & 0.496 & -0.13 & 0.617 & 0.05 & 0.844 & -0.08 & 0.767 \\
\hline \multirow[t]{4}{*}{ F major } & FA & 0.31 & 0.221 & 0.52 & $0.027^{*}$ & 0.05 & 0.859 & 0.28 & 0.268 & 0.05 & 0.852 & 0.17 & 0.499 \\
\hline & $\mathrm{MD}$ & 0.17 & 0.527 & -0.35 & 0.154 & -0.03 & 0.917 & -0.09 & 0.716 & 0.19 & 0.469 & 0.11 & 0.675 \\
\hline & $\mathrm{AD}$ & 0.44 & 0.075 & -0.10 & 0.703 & 0.03 & 0.895 & 0.01 & 0.982 & 0.30 & 0.235 & 0.19 & 0.460 \\
\hline & $\mathrm{RD}$ & -0.04 & 0.886 & -0.48 & $0.045^{*}$ & -0.07 & 0.801 & -0.16 & 0.539 & 0.09 & 0.730 & 0.00 & 0.998 \\
\hline
\end{tabular}

Units of axial diffusivity and radial diffusivity are $10^{-3} \mathrm{~mm}^{2} / \mathrm{s}$ for the mean and standard deviation. ${ }^{*} \mathrm{p}<0.05$ (significance level), ${ }^{* *} \mathrm{p}<0.004$ (significance level after Bonferroni correction). WISC-IV: Wechsler Intelligence Scale for Children-Fourth Edition, FA: fractional anisotropy, MD: mean diffusivity, AD: axial diffusivity, RD: radial diffusivity, ASD: autism spectrum disorder, TD: typically developing, SLF: superior longitudinal fasciculus, ILF: inferior longitudinal fasciculus, IFO: inferior fronto-occipital fasciculus, UNC: uncinate fasciculus, ATR: anterior thalamic radiation, F minor: forceps minor, F major: forceps major

nificant correlations were observed between the Comprehension scores on the WISC-IV and FA in the right IFO and left ATR, between the Comprehension scores and MD in the left ATR, and between the Comprehension scores and RD in the right IFO and left ATR. Non-significant correlations were also observed between the Matching Numbers scores on the DN-CAS and MD in the left UNC and F minor, between the Matching Numbers scores and $\mathrm{RD}$ in the F minor, and between the Sentence Questions scores on the DN-CAS and $\mathrm{RD}$ in the right ILF in the ASD group. In the TD group, the Comprehension scores on the WISC-IV were non-significantly correlated with MD in the F minor (Table 5 and 6).

\section{DISCUSSION}

In this study, we found 1) characteristic cognitive weakness, 2) reduced FA value in the F major, and 3) no specific differentiation for cognitive function associated with the specific tract in children with ASD. To our knowledge, the present study is the first to investigate the relationships between cognitionrelated white matter tracts and cognitive function in children with ASD using the DN-CAS and DTI tractography.

Participants in the ASD group scored poorly on the Comprehension, Coding, and Cancellation subtests of the WISCIV when compared with TD controls, consistent with the findings of previous studies. ${ }^{910}$ Furthermore, on the DN-CAS, the ASD group exhibited significant weakness on the Matching Numbers and Sentence Questions subtests when compared with the TD group. The Matching Numbers section assesses planning, processing speed, and working memory, while the Sentence Questions section assesses verbal comprehension and syntax. ${ }^{13,15}$ Many children with ASD exhibit impairments in language comprehension, planning, and processing speed. ${ }^{6,10,63}$ Therefore, these findings indicate that the DN-CAS appropriately reveals the cognitive impairments of children with highfunctioning ASD.

\section{Differences in DTI measurements between the ASD and TD groups}

In the present study, the FA values in the F major were significantly lower in the ASD group than in the TD group, consistent with the findings of a previous study. ${ }^{33}$ The $\mathrm{F}$ major is a commissural fiber tract that consists of the splenium of the corpus callosum and connects the occipital lobes. ${ }^{61,64}$ The F major plays roles in visuospatial cognition, ${ }^{45}$ processing speed, ${ }^{65}$ memory and executive function, ${ }^{66}$ and vocabulary and semantic processing. ${ }^{45,67}$ Previous studies have indicated that children with ASD exhibit impairments in processing speed, planning, and language comprehension, ${ }^{6,10,63}$ suggesting that alterations to the F major may play a role in these ASD-related impairments.

\section{Correlations between DTI measurements and cognitive function}

Our findings indicated that, in the ASD group, the Comprehension scores on the WISC-IV exhibited a positive correlation with $\mathrm{MD}$ in the F minor. Previous studies have revealed that the $\mathrm{F}$ minor plays a role in syntactic performance ${ }^{68}$ In one functional MRI study, activation of the left inferior frontal gyrus, including the pars orbitofrontal area, was lower in the ASD group than in the TD group during a sentence comprehension task. $^{69}$ Thus, our findings support the notion that the F minor plays a key role in comprehension, as assessed using the WISC- 

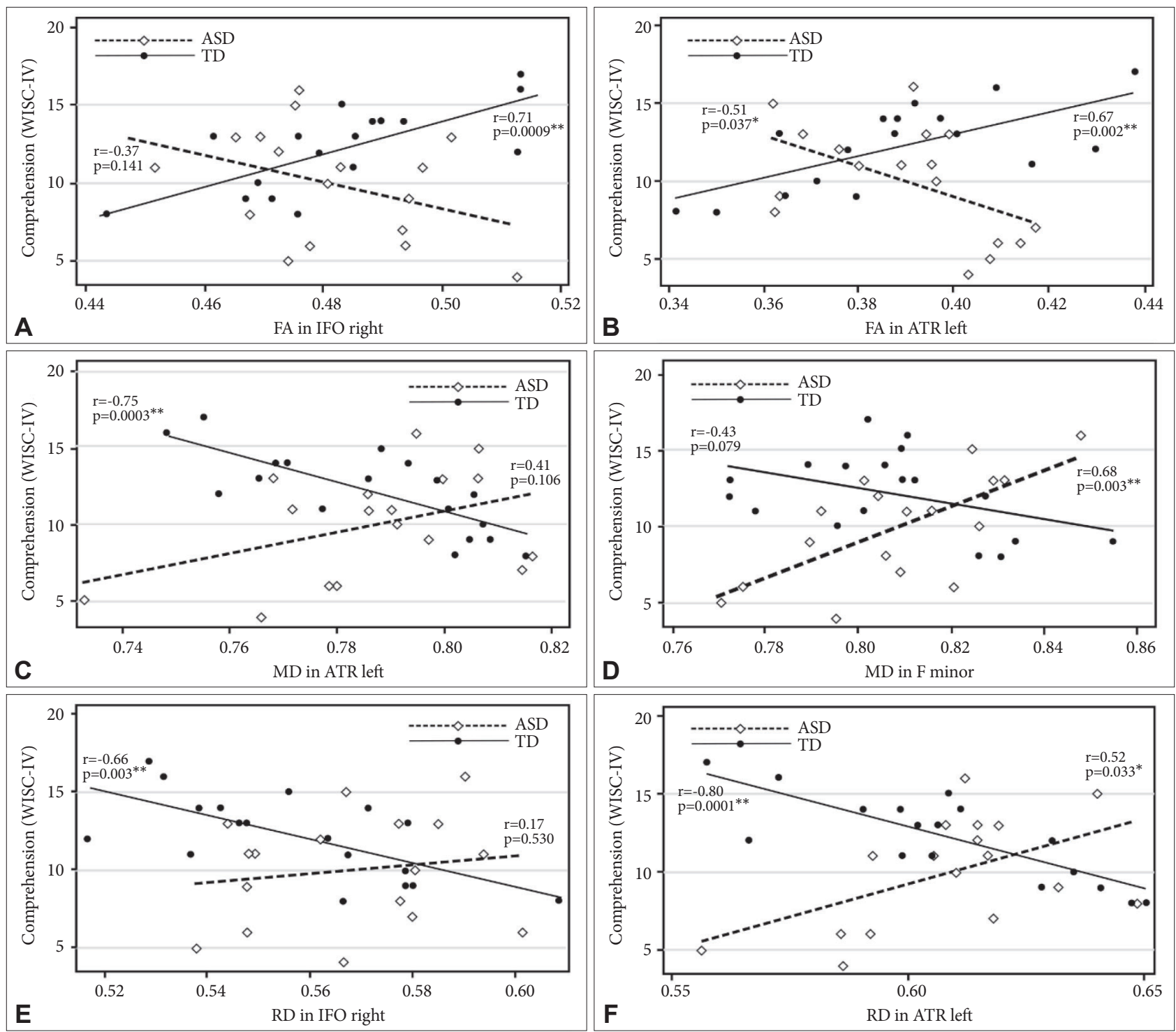

Figure 2. Scatter plots of the correlation analyses in the autism spectrum disorder (ASD) and typically developing (TD) groups. Scores on the Comprehension section of the Wechsler Intelligence Scale for Children-Fourth Edition (WISC-IV) are (A) positively correlated with fractional anisotropy (FA) values in the right inferior fronto-occipital fasciculus (IFO) in the TD group ( $r=0.71, p=0.0009)$, but not in the ASD group $(r=-0.37, p=0.141)$; (B) positively correlated with FA values in the left anterior thalamic radiation (ATR) in the TD group $(r=0.67, p=0.002)$, but not in the ASD group ( $r=-0.51, p=0.037)$; (C) negatively correlated with mean diffusivity (MD) values in the left ATR in the TD group ( $r=-0.75$, $p=0.0003)$, but not in the ASD group ( $r=0.41, p=0.106)$; (D) positively correlated with MD values in the forceps minor ( $F$ minor) in the ASD group ( $r=0.68, p=0.003$ ), but not in the TD group ( $r=0.43, p=0.079) ;(E)$ negatively correlated with radial diffusivity (RD) values in the right IFO in the TD group ( $r=-0.66, p=0.003)$, but not in the ASD group $(r=0.17, p=0.530)$; and $(F)$ negatively correlated with RD values in the left ATR in the TD group ( $r=-0.80, p=0.0001)$, but not in the ASD group $(r=0.52, p=0.033)$. Scores on the Matching Numbers portion of the Das-Naglieri Cognitive Assessment System (DN-CAS) are (G) positively correlated with MD values in the left uncinate fasciculus (UNC) in the TD group $(r=0.65, p=0.004)$, but not in the ASD group $(r=0.15, p=0.572) ;(H)$ positively correlated with MD values in the $F$ minor in the TD group $(r=0.67, p=0.003)$, but not in the ASD group $(r=-0.03, p=0.923)$; and (I) positively correlated with RD values in the $F$ minor in the TD group $(r=0.74, p=0.0004)$, but not in the ASD group ( $r=0.01, p=0.973)$. Scores on the Sentence Questions portion of the DN-CAS are $(J)$ positively correlated with RD in the right inferior longitudinal fasciculus (ILF) in the TD group ( $r=0.65, p=0.004)$, but not in the ASD group ( $r=0.02$, $p=0.934) .{ }^{*} p<0.05$ (significance level), ${ }^{* *} p<0.004$ (significance level following Bonferroni correction).

IV, among individuals with ASD.

Other than the aforementioned correlation, no correlations were observed between the subtest scores and DTI outcome measurements in the ASD group. In contrast, nine correlations between DTI measurements and the subtest scores on the WISC-IV and DN-CAS were observed in the TD group. It is known that the maturation of white matter tracts that connect restricted regions is closely associated with cognitive development in those particular regions. ${ }^{48}$ Therefore, our findings suggest that, in children with ASD, specific brain areas exhibit a weaker relationship with specific cognitive functions than they do in TD controls, resulting in less efficient cognitive pathways. 

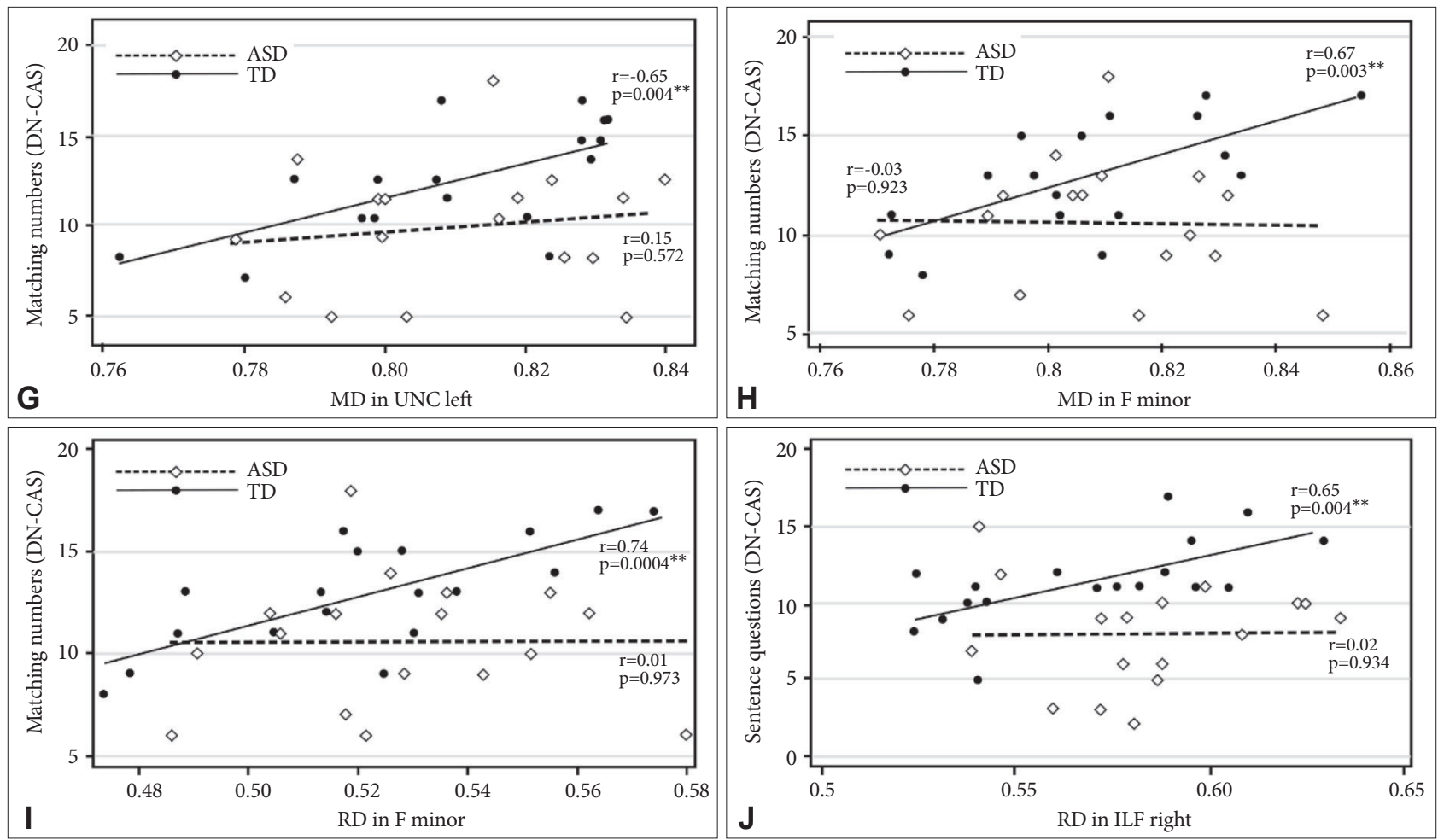

Figure 2. Scatter plots of the correlation analyses in the autism spectrum disorder (ASD) and typically developing (TD) groups. Scores on the Comprehension section of the Wechsler Intelligence Scale for Children-Fourth Edition (WISC-IV) are (A) positively correlated with fractional anisotropy (FA) values in the right inferior fronto-occipital fasciculus (IFO) in the TD group ( $r=0.71, p=0.0009)$, but not in the ASD group $(r=-0.37, p=0.141) ;(B)$ positively correlated with FA values in the left anterior thalamic radiation (ATR) in the TD group $(r=0.67, p=0.002)$, but not in the ASD group ( $r=-0.51, p=0.037)$; (C) negatively correlated with mean diffusivity (MD) values in the left ATR in the TD group ( $r=-0.75$, $p=0.0003)$, but not in the ASD group ( $r=0.41, p=0.106)$; $(D)$ positively correlated with MD values in the forceps minor ( $F$ minor) in the ASD group ( $r=0.68, p=0.003)$, but not in the TD group ( $r=0.43, p=0.079) ;(E)$ negatively correlated with radial diffusivity (RD) values in the right IFO in the TD group ( $r=-0.66, p=0.003)$, but not in the ASD group $(r=0.17, p=0.530)$; and $(F)$ negatively correlated with RD values in the left ATR in the TD group ( $r=-0.80, p=0.0001)$, but not in the ASD group $(r=0.52, p=0.033)$. Scores on the Matching Numbers portion of the Das-Naglieri Cognitive Assessment System (DN-CAS) are (G) positively correlated with MD values in the left uncinate fasciculus (UNC) in the TD group $(r=0.65, p=0.004)$, but not in the ASD group $(r=0.15, p=0.572) ;(H)$ positively correlated with MD values in the $F$ minor in the TD group $(r=0.67, p=0.003)$, but not in the ASD group $(r=-0.03, p=0.923)$; and $(I)$ positively correlated with RD values in the $F$ minor in the TD group $(r=0.74, p=0.0004)$, but not in the ASD group $(r=0.01, p=0.973)$. Scores on the Sentence Questions portion of the DN-CAS are $(J)$ positively correlated with RD in the right inferior longitudinal fasciculus (ILF) in the TD group $(r=0.65, p=0.004)$, but not in the ASD group ( $r=0.02$, $p=0.934) .{ }^{*} p<0.05$ (significance level), ${ }^{* *} p<0.004$ (significance level following Bonferroni correction).

Indeed, several functional MRI studies have demonstrated that the brains of individuals with ASD exhibit broader activation during various tasks. ${ }^{70,71}$ Although white matter maturation supports the development of cognitive function in TD children ${ }^{48}$ this effect is delayed in children with ASD. ${ }^{72}$ However, recent studies have revealed that adaptive myelination may occur, depending on the neuronal activity. Eigsti et al. ${ }^{73}$ reported that early intervention and experience may normalize behavioral language performance and that individuals with an optimal ASD outcome exhibit plasticity within the neural circuits associated with language.

More precisely, structural changes to white matter during childhood reflect the interplay among cell proliferation and apoptosis, dendritic branching and pruning, and synaptic formation and elimination. Therefore, such changes are influenced by the strength of proper neural connections and the pruning of inefficient pathways. ${ }^{74}$ However, further studies are required to determine whether early intervention during the critical period can alter the myelination trajectory of young children with ASD and whether such alterations can influence the acquisition of cognitive skills in the future.

The present study possesses several limitations of note. First, the sample was small and included only boys. Howe et al. ${ }^{75}$ reported that boys and girls with ASD exhibit differences in their cognitive abilities. Although the present study focused on boys alone, further studies focusing on the association between white matter tracts and cognition in girls with ASD are required. Second, to exclude the effects of age, sex, and IQ, we focused on children with high-functioning ASD. Therefore, our findings may not be applicable to children with low-functioning ASD. To determine whether our findings can be generalized to all individuals with ASD, a larger sample size in- 
Table 6. Correlations between the matching numbers and sentence questions scores on the DN-CAS and the FA, MD, AD, and RD values of white matter tracts

\begin{tabular}{|c|c|c|c|c|c|c|c|c|c|}
\hline & & \multicolumn{4}{|c|}{ Matching numbers } & \multicolumn{4}{|c|}{ Sentence questions } \\
\hline & & \multicolumn{2}{|c|}{$\operatorname{ASD}(\mathrm{N}=17)$} & \multicolumn{2}{|c|}{$\mathrm{TD}(\mathrm{N}=18)$} & \multicolumn{2}{|c|}{$\operatorname{ASD}(\mathrm{N}=17)$} & \multicolumn{2}{|c|}{$\mathrm{TD}(\mathrm{N}=18)$} \\
\hline & & rvalue & $\mathrm{p}$ value & r value & $\mathrm{p}$ value & r value & $\mathrm{p}$ value & r value & $\mathrm{p}$ value \\
\hline \multirow[t]{4}{*}{ SLF left } & FA & 0.12 & 0.639 & -0.35 & 0.158 & -0.36 & 0.159 & -0.15 & 0.551 \\
\hline & $\mathrm{MD}$ & -0.29 & 0.263 & 0.50 & $0.033^{*}$ & -0.01 & 0.801 & 0.49 & $0.041^{*}$ \\
\hline & $\mathrm{AD}$ & -0.18 & 0.498 & 0.32 & 0.191 & -0.31 & 0.231 & 0.42 & 0.079 \\
\hline & $\mathrm{RD}$ & -0.27 & 0.296 & 0.49 & $0.040^{*}$ & 0.18 & 0.495 & 0.39 & 0.113 \\
\hline \multirow[t]{4}{*}{ SLF right } & FA & -0.20 & 0.446 & -0.41 & 0.092 & -0.01 & 0.975 & -0.15 & 0.561 \\
\hline & $\mathrm{MD}$ & -0.03 & 0.915 & 0.37 & 0.131 & -0.01 & 0.980 & 0.48 & $0.043^{*}$ \\
\hline & $\mathrm{AD}$ & -0.14 & 0.596 & 0.14 & 0.586 & 0.00 & 0.989 & 0.43 & 0.073 \\
\hline & $\mathrm{RD}$ & 0.07 & 0.783 & 0.42 & 0.082 & -0.01 & 0.961 & 0.35 & 0.149 \\
\hline \multirow[t]{4}{*}{ ILF left } & FA & 0.43 & 0.084 & -0.57 & $0.014^{*}$ & -0.21 & 0.422 & -0.31 & 0.206 \\
\hline & $\mathrm{MD}$ & -0.03 & 0.924 & 0.52 & $0.027^{*}$ & 0.09 & 0.744 & 0.58 & $0.011^{*}$ \\
\hline & $\mathrm{AD}$ & 0.26 & 0.308 & 0.12 & 0.628 & -0.02 & 0.948 & 0.47 & $0.049^{*}$ \\
\hline & $\mathrm{RD}$ & -0.28 & 0.282 & 0.58 & $0.011^{*}$ & 0.15 & 0.575 & 0.49 & $0.039^{*}$ \\
\hline \multirow[t]{4}{*}{ ILF right } & FA & 0.15 & 0.572 & -0.50 & $0.033^{*}$ & 0.07 & 0.799 & -0.60 & $0.008^{*}$ \\
\hline & $\mathrm{MD}$ & -0.28 & 0.282 & 0.56 & $0.017^{*}$ & 0.14 & 0.589 & 0.62 & $0.006^{*}$ \\
\hline & $\mathrm{AD}$ & -0.15 & 0.561 & 0.20 & 0.437 & 0.22 & 0.403 & 0.18 & 0.465 \\
\hline & $\mathrm{RD}$ & -0.22 & 0.394 & 0.57 & $0.013^{*}$ & 0.02 & 0.934 & 0.65 & $0.004^{* *}$ \\
\hline \multirow[t]{4}{*}{ IFO left } & FA & 0.44 & 0.075 & -0.62 & $0.006^{*}$ & -0.05 & 0.859 & -0.24 & 0.346 \\
\hline & $\mathrm{MD}$ & -0.12 & 0.633 & 0.34 & 0.189 & 0.21 & 0.412 & 0.58 & $0.011^{*}$ \\
\hline & $\mathrm{AD}$ & 0.20 & 0.443 & -0.20 & 0.419 & 0.17 & 0.524 & 0.38 & 0.117 \\
\hline & $\mathrm{RD}$ & -0.36 & 0.160 & 0.55 & $0.017^{*}$ & 0.18 & 0.495 & 0.48 & $0.042^{*}$ \\
\hline \multirow[t]{4}{*}{ IFO right } & FA & 0.24 & 0.360 & -0.22 & 0.373 & -0.23 & 0.370 & -0.25 & 0.315 \\
\hline & $\mathrm{MD}$ & -0.28 & 0.270 & 0.52 & $0.026^{*}$ & -0.05 & 0.837 & 0.62 & $0.006^{*}$ \\
\hline & $\mathrm{AD}$ & -0.18 & 0.500 & 0.45 & 0.062 & -0.16 & 0.537 & 0.56 & $0.015^{*}$ \\
\hline & $\mathrm{RD}$ & -0.34 & 0.177 & 0.44 & 0.069 & 0.07 & 0.789 & 0.50 & $0.034^{*}$ \\
\hline \multirow[t]{4}{*}{ UNC left } & FA & -0.09 & 0.726 & -0.44 & 0.071 & -0.27 & 0.291 & -0.42 & 0.082 \\
\hline & $\mathrm{MD}$ & 0.15 & 0.572 & 0.65 & $0.004^{* *}$ & -0.04 & 0.889 & 0.60 & $0.009^{*}$ \\
\hline & $\mathrm{AD}$ & 0.06 & 0.816 & 0.47 & $0.047^{*}$ & -0.22 & 0.389 & 0.47 & $0.047^{*}$ \\
\hline & $\mathrm{RD}$ & 0.17 & 0.520 & 0.59 & $0.009^{*}$ & 0.09 & 0.733 & 0.53 & $0.023^{*}$ \\
\hline \multirow[t]{4}{*}{ UNC right } & FA & -0.08 & 0.772 & -0.37 & 0.130 & -0.02 & 0.943 & -0.44 & 0.071 \\
\hline & $\mathrm{MD}$ & -0.10 & 0.705 & 0.50 & $0.034^{*}$ & -0.18 & 0.484 & 0.56 & $0.017^{*}$ \\
\hline & $\mathrm{AD}$ & -0.14 & 0.583 & 0.38 & 0.124 & -0.24 & 0.358 & 0.43 & 0.072 \\
\hline & $\mathrm{RD}$ & -0.05 & 0.840 & 0.48 & $0.043^{*}$ & -0.11 & 0.663 & 0.53 & $0.024^{*}$ \\
\hline \multirow[t]{4}{*}{ ATR left } & FA & -0.34 & 0.181 & -0.49 & $0.041^{*}$ & -0.65 & $0.005^{*}$ & -0.37 & 0.127 \\
\hline & $\mathrm{MD}$ & 0.15 & 0.569 & 0.40 & 0.096 & 0.06 & 0.817 & 0.50 & $0.036^{*}$ \\
\hline & $\mathrm{AD}$ & -0.15 & 0.577 & -0.06 & 0.799 & -0.39 & 0.121 & 0.20 & 0.423 \\
\hline & $\mathrm{RD}$ & 0.31 & 0.228 & 0.50 & $0.033^{*}$ & 0.35 & 0.165 & 0.48 & $0.042^{*}$ \\
\hline \multirow[t]{4}{*}{ ATR right } & FA & 0.01 & 0.962 & -0.03 & 0.901 & -0.27 & 0.302 & -0.20 & 0.427 \\
\hline & $\mathrm{MD}$ & 0.04 & 0.883 & 0.00 & 0.990 & 0.33 & 0.200 & 0.40 & 0.099 \\
\hline & $\mathrm{AD}$ & 0.04 & 0.870 & -0.08 & 0.765 & 0.09 & 0.726 & 0.31 & 0.208 \\
\hline & $\mathrm{RD}$ & 0.02 & 0.933 & 0.05 & 0.833 & 0.38 & 0.132 & 0.38 & 0.117 \\
\hline
\end{tabular}


Table 6. Correlations between the matching numbers and sentence questions scores on the DN-CAS and the FA, MD, AD, and RD values of white matter tracts (contiued)

\begin{tabular}{|c|c|c|c|c|c|c|c|c|c|}
\hline & & \multicolumn{4}{|c|}{ Matching numbers } & \multicolumn{4}{|c|}{ Sentence questions } \\
\hline & & \multicolumn{2}{|c|}{$\operatorname{ASD}(\mathrm{N}=17)$} & \multicolumn{2}{|c|}{$\mathrm{TD}(\mathrm{N}=18)$} & \multicolumn{2}{|c|}{$\operatorname{ASD}(\mathrm{N}=17)$} & \multicolumn{2}{|c|}{$\mathrm{TD}(\mathrm{N}=18)$} \\
\hline & & r value & $\mathrm{p}$ value & r value & $\mathrm{p}$ value & rvalue & $\mathrm{p}$ value & r value & $\mathrm{p}$ value \\
\hline \multirow[t]{4}{*}{ F minor } & FA & -0.03 & 0.896 & -0.61 & $0.008^{*}$ & -0.23 & 0.379 & -0.52 & $0.028^{*}$ \\
\hline & $\mathrm{MD}$ & -0.03 & 0.923 & 0.67 & $0.003^{* *}$ & 0.21 & 0.424 & 0.40 & 0.102 \\
\hline & $\mathrm{AD}$ & -0.08 & 0.752 & 0.06 & 0.819 & 0.11 & 0.672 & -0.10 & 0.686 \\
\hline & $\mathrm{RD}$ & 0.01 & 0.973 & 0.74 & $0.0004^{* *}$ & 0.20 & 0.440 & 0.51 & $0.029^{*}$ \\
\hline \multirow[t]{4}{*}{ F major } & FA & 0.42 & 0.092 & -0.22 & 0.384 & -0.11 & 0.670 & -0.19 & 0.451 \\
\hline & $\mathrm{MD}$ & -0.34 & 0.181 & -0.01 & 0.975 & 0.34 & 0.178 & 0.34 & 0.167 \\
\hline & $\mathrm{AD}$ & -0.22 & 0.397 & -0.15 & 0.547 & 0.43 & 0.085 & 0.25 & 0.311 \\
\hline & $\mathrm{RD}$ & -0.39 & 0.125 & 0.13 & 0.612 & 0.25 & 0.334 & 0.32 & 0.200 \\
\hline
\end{tabular}

Units of axial diffusivity and radial diffusivity are $10^{-3} \mathrm{~mm}^{2} / \mathrm{s}$ for the mean and standard deviation. ${ }^{*} \mathrm{p}<0.05$ (significance level), ${ }^{* *} \mathrm{p}<0.004$ (significance level after Bonferroni correction). DN-CAS: Das-Naglieri Cognitive Assessment System, FA: fractional anisotropy, MD: mean diffusivity, AD: axial diffusivity, RD: radial diffusivity, ASD: autism spectrum disorder, TD: typically developing, SLF: superior longitudinal fasciculus, ILF: inferior longitudinal fasciculus, IFO: inferior fronto-occipital fasciculus, UNC: uncinate fasciculus, ATR: anterior thalamic radiation, F minor: forceps minor, F major: forceps major

cluding individuals with a broader range of IQs is required. Third, in the correlation analysis, our results for $\mathrm{MD}$ and $\mathrm{RD}$ were inconsistent. Although MD and RD decrease with development, we could not analyze longitudinal changes in cognitive functions and white matter microstructure due to the narrow age range of the participants.

In conclusion, the results of the present study demonstrate that children with ASD and TD controls exhibit significant differences in cognitive functions such as language comprehension, processing speed, and planning, and in the white matter microstructure of the F major. Moreover, although many correlations were observed between DTI measurements and subscale scores in the TD group, only one correlation was observed in the ASD group. Thus, our findings suggest that, in individuals with ASD, processing skills depend on a widespread neural network that is less directly associated with specific tract measurements, and that the specific brain areas affected are less efficiently engaged in their respective cognitive pathways. In addition, our findings may aid in clarifying the mechanisms underlying cognitive dysfunction in children with ASD and provide evidence in support of early intervention for their cognitive and social development.

\section{Acknowledgments}

This study was supported by JSPS KAKENHI Grant numbers 15K09619 and 15H01581 and the Program for Promoting the Enhancement of Research Universities by the Ministry of Education, Culture, Sports, Science and Technology. We wish to thank all the children and their parents for their participation in this study.

\section{REFERENCES}

1. American Psychiatric Association. Diagnostic and Statistical Manual of Mental Disorders, DSM-5. Washington, DC: American Psychiatric Association; 2013.

2. Lai MC, Lombardo MV, Baron-Cohen S. Autism. Lancet 2014;383:896910.

3. Durrleman S, Hippolyte L, Zufferey S, Iglesias K, Hadjikhani N. Complex syntax in autism spectrum disorders: a study of relative clauses. Int J Lang Commun Disord 2015;50:260-267.

4. Ozonoff S, Pennington BF, Rogers SJ. Executive function deficits in high-functioning autistic individuals: relationship to theory of mind. J Child Psychol Psychiatry 1991;32:1081-1105.

5. Hill EL. Executive dysfunction in autism. Trends Cogn Sci 2004;8:2632.

6. Sinzig J, Morsch D, Bruning N, Schmidt MH, Lehmkuhl G. Inhibition, flexibility, working memory and planning in autism spectrum disorders with and without comorbid ADHD-symptoms. Child Adolesc Psychiatry Ment Health 2008;2:4.

7. Steele SD, Minshew NJ, Luna B, Sweeney JA. Spatial working memory deficits in autism. J Autism Dev Disord 2007;37:605-612.

8. Mottron L. Matching strategies in cognitive research with individuals with high-functioning autism: current practices, instrument biases, and recommendations. J Autism Dev Disord 2004;34:19-27.

9. Mayes SD, Calhoun SL. WISC-IV and WIAT-II profiles in children with high-functioning autism. J Autism Dev Disord 2008;38:428-439.

10. Oliveras-Rentas RE, Kenworthy L, Roberson RB, Martin A, Wallace GL. WISC-IV profile in high-functioning autism spectrum disorders: impaired processing speed is associated with increased autism communication symptoms and decreased adaptive communication abilities. J Autism Dev Disord 2012;42:655-664.

11. Nader AM, Jelenic P, Soulières I. Discrepancy between WISC-III and WISC-IV cognitive profile in autism spectrum: what does it reveal about autistic cognition? PLoS ONE 2015;10:e0144645.

12. Das JP, Naglieri JA, Kirby JR. Assessment of Cognitive Processes: the PASS Theory of Intelligence. Boston, MA: Allyn and Bacon; 1994.

13. Naglieri JA, Das JP. Cognitive Assessment System. Itasca, IL: Riverside Publishing; 1997.

14. Naglieri JA. Essentials of CAS Assessment. New York, NY: John Wiley and Sons; 1999.

15. McCrea SM. A cognitive neuropsychological examination of the DasNaglieri Cognitive Assessment System subtests: a report of three stroke 
cases studied longitudinally during recovery. Int J Neurosci 2009;119: 553-599.

16. Taddei S, Contena B. Brief Report: Cognitive performance in autism and Asperger's syndrome: what are the differences? J Autism Dev Disord 2013;43:2977-2983.

17. Le Bihan D. Looking into the functional architecture of the brain with diffusion MRI. Nat Rev Neurosci 2003;4:469-480.

18. Mori S, Zhang J. Principles of diffusion tensor imaging and its applications to basic neuroscience research. Neuron 2006;51:527-539.

19. Le Bihan D, Johansen-Berg H. Diffusion MRI at 25: exploring brain tissue structure and function. Neuroimage 2012;61:324-341.

20. Beaulieu C. The basis of anisotropic water diffusion in the nervous system - a technical review. NMR Biomed 2002;15:435-455.

21. Feldman HM, Yeatman JD, Lee ES, Barde LHF, Gaman-Bean S. Diffusion tensor imaging: a review for pediatric researchers and clinicians. J Dev Behav Pediatr 2010;31:346-356.

22. Alexander AL, Hurley SA, Samsonov AA, Adluru N, Hosseinbor AP, Mossahebi $\mathrm{P}$, et al. Characterization of cerebral white matter properties using quantitative magnetic resonance imaging stains. Brain Connect 2011;1:423-446.

23. Tournier JD, Mori S, Leemans A. Diffusion tensor imaging and beyond. Magn Reson Med 2011;65:1532-1556.

24. Basser PJ, Mattiello J, Lebihan D. Estimation of the effective self-diffusion tensor from the NMR spin echo. J Magn Reson B 1994;103:247-254.

25. Pierpaoli C, Jezzard P, Basser PJ, Barnett A, Chiro GD. Diffusion tensor MR imaging of the human brain. Radiology 1996;201:637-648.

26. Sbardella E, Tona F, Petsas N, Pantano P. DTI measurements in multiple sclerosis: evaluation of brain damage and clinical implications. Mult Scler Int 2013;2013:671730.

27. Song SK, Sun SW, Ramsbottom MJ, Chang C, Russell J, Cross AH. Dysmyelination revealed through MRI as increased radial (but unchanged axial) diffusion of water. Neuroimage 2002;17:1429-1436.

28. Wu YC, Field AS, Duncan ID, Samsonov AA, Kondo Y, Tudorascu D, et al. High b-value and diffusion tensor imaging in a canine model of dysmyelination and brain maturation. Neuroimage 2011;58:829-837.

29. Huang H, Zhang J, van Zijl PC, Mori S. Analysis of noise effects on DTIbased tractography using the brute-force and multi-ROI approach. Magn Reson Med 2004;52:559-565.

30. Froeling M, Pullens P, Leemans A. DTI Analysis Methods: Region of Interest Analysis. In: Van Hecke W, Emsell L, Sunaert S, Editors. Diffusion Tensor Imaging: A Practical Handbook. New York, NY: Springer New York, 2016, p. 175-182.

31. Smith SM, Jenkinson M, Johansen-Berg H, Rueckert D, Nichols TE, Mackay CE, et al. Tract-based spatial statistics: voxelwise analysis of multi-subject diffusion data. Neuroimage 2006;31:1487-1505.

32. Cheon KA, Kim YS, Oh SH, Park SY, Yoon HW, Herrington J, et al. Involvement of the anterior thalamic radiation in boys with high functioning autism spectrum disorders: a diffusion tensor imaging study. Brain Res 2011;1417:77-86.

33. Shukla DK, Keehn B, Müller RA. Tract-specific analyses of diffusion tensor imaging show widespread white matter compromise in autism spectrum disorder. J Child Psychol Psychiatry 2011;52:286-295.

34. Bakhtiari R, Zürcher NR, Rogier O, Russo B, Hippolyte L, Granziera C, et al. Differences in white matter reflect atypical developmental trajectory in autism: a tract-based spatial statistics study. Neuroimage Clin 2012;1:48-56.

35. Chang YS, Owen JP, Desai SS, Hill SS, Arnett AB, Harris J, et al. Autism and sensory processing disorders: shared white matter disruption in sensory pathways but divergent connectivity in social-emotional pathways. PLoS ONE 2014;9:e103038.

36. Roine U, Salmi J, Roine T, Wendt TN, Leppämäki S, Rintahaka P, et al. Constrained spherical deconvolution-based tractography and tract-based spatial statistics show abnormal microstructural organization in Asperger syndrome. Mol Autism 2015;6:4.

37. Catani M, Dell'Acqua F, Budisavljevic S, Howells H, Thiebaut de Schot- ten M, Froudist-Walsh S, et al. Frontal networks in adults with autism spectrum disorder. Brain 2016;139:616-630.

38. Bendlin BB, Fitzgerald ME, Ries ML, Xu G, Kastman EK, Thiel BW, et al. White matter in aging and cognition: a cross-sectional study of microstructure in adults aged eighteen to eighty-three. Dev Neuropsychol 2010;35:257-277.

39. Madhavan KM, McQueeny T, Howe SR, Shear P, Szaflarski J. Superior longitudinal fasciculus and language functioning in healthy aging. Brain Res 2014;1562:11-22.

40. Wong FCK, Chandrasekaran B, Garibaldi K, Wong PCM. White matter anisotropy in the ventral language pathway predicts sound-to-word learning success. J Neurosci 2011;31:8780-8785.

41. de Zubicaray GI, Rose SE, McMahon KL. The structure and connectivity of semantic memory in the healthy older adult brain. Neuroimage 2011;54:1488-1494.

42. Mabbott DJ, Rovet J, Noseworthy MD, Smith ML, Rockel C. The relations between white matter and declarative memory in older children and adolescents. Brain Res 2009;1294:80-90.

43. Martínez K, Merchán-Naranjo J, Pina-Camacho L, Alemán-Gómez Y, Boada L, Fraguas D, et al. Atypical age-dependency of executive function and white matter microstructure in children and adolescents with autism spectrum disorders. Eur Child Adolesc Psychiatry 2017;26:13611376.

44. Zahr NM, Rohlfing T, Pfefferbaum A, Sullivan EV. Problem solving, working memory, and motor correlates of association and commissural fiber bundles in normal aging: a quantitative fiber tracking study. Neuroimage 2009;44:1050-1062.

45. Fryer SL, Frank LR, Spadoni AD, Theilmann RJ, Nagel BJ, Schweinsburg $\mathrm{AD}$, et al. Microstructural integrity of the corpus callosum linked with neuropsychological performance in adolescents. Brain Cogn 2008;67: 225-233.

46. Alexander AL, Lee JE, Lazar M, Boudos R, DuBray MB, Oakes TR, et al. Diffusion tensor imaging of the corpus callosum in Autism. Neuroimage 2007;34:61-73.

47. Hanaie R, Mohri I, Kagitani-Shimono K, Tachibana M, Matsuzaki J, Watanabe Y, et al. Abnormal corpus callosum connectivity, socio-communicative deficits, and motor deficits in children with autism spectrum disorder: a diffusion tensor imaging study. J Autism Dev Disord 2014; 44:2209-2220.

48. Nagy Z, Westerberg H, Klingberg T. Maturation of white matter is associated with the development of cognitive functions during childhood. J Cogn Neurosci 2004;16:1227-1233.

49. Yeatman JD, Wandell BA, Mezer AA. Lifespan maturation and degeneration of human brain white matter. Nat Commun 2014;5:4932-4932.

50. Oldfield RC. The assessment and analysis of handedness: the Edinburgh inventory. Neuropsychologia 1971;9:97-113.

51. Lord C, Risi S, Lambrecht L, Cook EH, Leventhal BL, DiLavore PC, et al. The autism diagnostic observation schedule-generic: a standard measure of social and communication deficits associated with the spectrum of autism. J Autism Dev Disord 2000;30:205-223.

52. Berument SK, Rutter M, Lord C, Pickles A, Bailey A. Autism screening questionnaire: diagnostic validity. Br J Psychiatry 1999;175:444-451.

53. Dairoku H, Senju A, Hayashi E, Tojo Y, Ichikawa H. Development of Japanese version of autism screening questionnaire. Kokuritsu Tokushu Kyoiku Kenkyusho Ippan Kenkyu Houkokusho 2004;7:19-34.

54. Wechsler D. Wechsler Intelligence Scale for Children-Fourth Edition (WISC-IV). San Antonio, TX: The Psychological Corporation; 2003.

55. Flanagan DP, Kaufman AS. Essentials of WISC-IV Assessment, Second Edition. Hoboken, NJ: John Wiley \& Sons International; 2009.

56. Maekawa H, Nakayama T, Okazaki S. The Das-Naglieri Cognitive Assessment System [Japanese Adaptation]. Tokyo: Nihon Bunka Kagakusha; 2007.

57. Leemans A, Jones DK. The B-matrix must be rotated when correcting for subject motion in DTI data. Magn Reson Med 2009;61:1336-1349.

58. Basser PJ, Pajevic S, Pierpaoli C, Duda J, Aldroubi A. In vivo fiber trac- 
tography using DT-MRI data. Magn Reson Med 2000;44:625-632.

59. Jeurissen B, Leemans A, Jones DK, Tournier JD, Sijbers J. Probabilistic fiber tracking using the residual bootstrap with constrained spherical deconvolution. Hum Brain Mapp 2011;32:461-479.

60. Wakana S, Caprihan A, Panzenboeck MM, Fallon JH, Perry M, Gollub RL, et al. Reproducibility of quantitative tractography methods applied to cerebral white matter. Neuroimage 2007;36:630-644.

61. Catani M, Howard RJ, Pajevic S, Jones DK. Virtual in vivo interactive dissection of white matter fasciculi in the human brain. Neuroimage 2002; 17:77-94.

62. StataCorp. Stata Statistical Software: Release 14. College Station, TX: StataCorp LP; 2015.

63. Kover ST, Haebig E, Oakes A, McDuffie A, Hagerman RJ, Abbeduto L. Sentence comprehension in boys with autism spectrum disorder. Am J Speech Lang Pathol 2014;23:385-394.

64. Fitsiori A, Nguyen D, Karentzos A, Delavelle J, Vargas MI. The corpus callosum: white matter or terra incognita. Br J Radiol 2011;84:5-18.

65. Kerchner GA, Racine CA, Hale S, Wilheim R, Laluz V, Miller BL, et al. Cognitive processing speed in older adults: relationship with white matter integrity. PLoS ONE 2012;7:e50425.

66. Voineskos AN, Rajji TK, Lobaugh NJ, Miranda D, Shenton ME, Kennedy JL, et al. Age-related decline in white matter tract integrity and cognitive performance: a DTI tractography and structural equation modeling study. Neurobiol Aging 2012;33:21-34.

67. Saur D, Schelter B, Schnell S, Kratochvil D, Küpper H, Kellmeyer P, et al. Combining functional and anatomical connectivity reveals brain net- works for auditory language comprehension. Neuroimage 2010;49:31873197.

68. Antonenko D, Brauer J, Meinzer M, Fengler A, Kerti L, Friederici AD, et al. Functional and structural syntax networks in aging. Neuroimage 2013; 83:513-523.

69. Just MA, Cherkassky VL, Keller TA, Minshew NJ. Cortical activation and synchronization during sentence comprehension in high-functioning autism: evidence of underconnectivity. Brain 2004;127:1811-1821.

70. Wang AT, Lee SS, Sigman M, Dapretto M. Neural basis of irony comprehension in children with autism: the role of prosody and context. Brain 2006;129:932-943.

71. Knaus TA, Silver AM, Lindgren KA, Hadjikhani N, Tager-Flusberg H. fMRI activation during a language task in adolescents with ASD. J Int Neuropsychol Soc 2008;14:967-979.

72. Luna B, Doll SK, Hegedus SJ, Minshew NJ, Sweeney JA. Maturation of executive function in autism. Biol Psychiatry 2007;61:474-481.

73. Eigsti IM, Stevens MC, Schultz RT, Barton M, Kelley E, Naigles L, et al. Language comprehension and brain function in individuals with an optimal outcome from autism. Neuroimage Clin 2016;10:182-191.

74. Andersen SL. Trajectories of brain development: point of vulnerability or window of opportunity? Neurosci Biobehav Rev 2003;27:3-18.

75. Howe YJ, O’Rourke JA, Yatchmink Y, Viscidi EW, Jones RN, Morrow EM. Female autism phenotypes investigated at different levels of language and developmental abilities. J Autism Dev Disord 2015;45:35373549 . 